\title{
OBTENCIÓN DE QUITINA A PARTIR DEL EXOESQUELETO DE CAMARÓN (Litopenaeus vannamei) \\ COMO COAGULANTE - FLOCULANTE EN AGUAS DE BAJA TURBIDEZ
}

\section{OBTAINING CHITIN FROM THE SHRIMP (Litopenaeus vannmei) EXOSKELETON AS COAGULANT - FLOCCULANT IN WATERS OF LOW TURBIDITY}

\section{José Rosero ${ }^{1 *}$, Martha Suárez², Stephanie Mora ${ }^{3}$, Bryan Achig² $^{2}$ Adriana Chamba ${ }^{2} \&$ Alex Anrango $^{1}$}

\author{
Recibido: 4 de agosto 2019 / Aceptado: 10 de junio 2020 \\ Publicado en línea: 22 de junio 2020 \\ DOI: $10.26807 /$ ia.v8i2.113
}

\begin{abstract}
Palabras clave: biopolímero, exoesqueleto de camarón, coagulante, floculante, quitina, turbidez

Keywords: biopolymer, shrimp exoskeleton, coagulant, flocculant, chitin, turbidity.
\end{abstract}

1 Universidad Central del Ecuador. Facultad de Ingeniería Ciencias Físicas y Matemáticas. Carrera de Ingeniería Civil. Quito, Ecuador. (*correspondencia: jarosero@uce.edu.ec; anrango.alex@yahoo.es)

2 Universidad Central del Ecuador. Facultad de Ciencias Químicas. Carrera de Química. Quito, Ecuador. (masuarez@uce.edu.ec; andresmh2801@hotmail.com; aechamba@uce.edu.ec)

3 Universidad Central del Ecuador. Facultad de Ciencias Químicas. Carrera de Química Farmacéutica. Quito, Ecuador. (stepha_tai_13@hotmail.com) 


\section{RESUMEN}

Se obtuvo quitina a partir del exoesqueleto de camarón, con un rendimiento del 27,5\%, identificándose los valores promedio para desmineralización y desproteinización $97,80 \pm 3,13 \%$ (p/p) y 32,18 \pm 0,14 \% (p/p), respectivamente. El biopolímero obtenido se caracterizó por espectroscopia infrarroja con la presencia de bandas de estiramiento de los enlaces $-\mathrm{OH}$ a $3386 \mathrm{~cm}^{-1},-\mathrm{NH}$ a 3124 $\mathrm{cm}^{-1},-\mathrm{C}=\mathrm{O}$ a $1644 \mathrm{~cm}^{-1}$ además de las absorciones características de enlaces $-\mathrm{C}_{\mathrm{sp} 3} \mathrm{H}$ de la cadena carbonada, mismas que coinciden con la estructura de la unidad repetitiva del biopolímero. La quitina se obtuvo como un sólido de color blanco en forma de láminas, insoluble en agua y en ácidos diluidos, soluble en ácido clorhídrico concentrado y con un peso molecular de 202,978 \pm 1,235 g/mol. El polímero, se comporta como coagulante-floculante de sólidos suspendidos en agua, produciendo reducción en los niveles de turbidez. Con concentraciones experimentales entre 0,15-0,45 ppm de quitina, se observan descensos en la turbidez que de acuerdo a los correspondientes cálculos teóricos se encuentran entre 7,55-50,27\%; obteniéndose el valor máximo de $55,84 \%$ en el descenso de turbidez con 0,37 ppm de quitina. Así, este biopolímero se comporta como coagulante-floculante y produce reducción en los niveles de turbidez, esta característica lo convierte en una alternativa válida para disminuir el uso de compuestos de probada toxicidad en el tratamiento de aguas superficiales para consumo humano.

\section{ABSTRACT}

Chitin was obtained from shrimp exoskeleton, with a yield of $27.5 \%$, identifying the average values for demineralization and deproteinization with a weight percentage of $97.80 \pm 3.13 \%$ and $32.18 \pm 0.14 \%$ respectively. The biopolymer obtained was characterized by infrared spectroscopy with the presence of link stretch bands $-\mathrm{OH}$ at $3386 \mathrm{~cm}^{-1},-\mathrm{NH}$ at $3124 \mathrm{~cm}^{-1},-\mathrm{C}=\mathrm{O}$ at $1644 \mathrm{~cm}^{-1}$ in addition to the characteristic absorptions of bonds $-\mathrm{C}_{\mathrm{sp} 3} \mathrm{H}$ of the carbon chain, which match the structure of the repeating unit of the biopolymer. Chitin was obtained as a white solid in the form of sheets, insoluble in water and diluted 
acids, soluble in concentrated hydrochloric acid and with a molecular weight of $202.978 \pm 1.235 \mathrm{~g} / \mathrm{mol}$. The polymer behaves as a coagulant-flocculant of solids suspended in water, producing a reduction of turbidity levels. In experimental concentrations between 0.15-0.45 ppm of chitin, decreases in turbidity are observed, which according to the corresponding theoretical calculations are between 7.55-50.27\%; obtaining the maximum value of $55.84 \%$ in the decrease of turbidity with 0.37 ppm of chitin. Thus, this biopolymer behaves as coagulant-flocculant and produces reduction in turbidity levels; this characteristic makes it a valid alternative to reduce the use of compounds of proven toxicity in the treatment of surface waters for human consumption.

\section{INTRODUCCIÓN}

La industria camaronera de Ecuador procesa el camarón de tal manera que utiliza únicamente las colas para su exportación. La parte desechada es el cefalotórax. En promedio la composición del cefalotórax de camarón contiene quitina $27 \%$, proteína $40 \%$, carbonato de calcio 30 $\%$ y carotenos $3 \%$. Todos estos elementos son considerados materias primas renovables con un amplio potencial industrial, principalmente la quitina (Figura 1) que es el segundo polímero natural más abundante después de la celulosa y está formado por unidades de N-acetil-D-glucosamina (Restrepo \& Guarín, 2004).

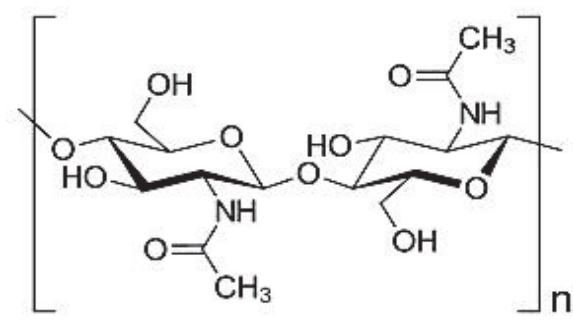

Figura 1. Unidades repetitivas de quitina

(Velásquez, 2003)

El proceso de obtención de quitina consiste en lograr la desmineralización-desproteinización del exoesqueleto de camarón, después de lo cual se obtiene una amida insoluble en agua y en ácidos diluidos (Wade, 2012). 
La caracterización de la quitina se realiza mediante la determinación de su peso molecular medio y del contenido de nitrógeno; así como también mediante la identificación de las bandas de absorción de los grupos amido mediante espectroscopia infrarroja.

A partir de la quitina se pueden obtener derivados solubles en agua como: acetatos, cloruros y sulfatos, por tratamiento de ésta con ácidos (López, 2012), los mismos que podrían ser utilizados en procesos de potabilización de agua para consumo humano (Velásquez, 2003).

El peso molecular de la quitina está directamente relacionado con el tamaño del biopolímero, -aspecto que facilitará su sedimentación en aguamientras que el contenido de nitrógeno se relaciona con la posibilidad de generar puntos catiónicos en la molécula, los cuales desestabilizan la carga superficial de los coloides.

El proceso central en el tratamiento de aguas crudas para consumo humano -en su mayoría aguas superficiales- consiste en la remoción de materiales de naturaleza coloidal tales como arcilla, limo y lodos. La eliminación de los materiales remanentes se logra con la utilización de coagulantes, así como de coadyuvantes de floculación.

Tradicionalmente los productos que se han usado con mayor frecuencia son sales metálicas tales como sulfato de aluminio, cloruro férrico y sulfato ferroso.

La coagulación es el método que permite la remoción de partículas coloidales y suspendidas, durante el proceso de clarificación, además de que reduce la turbidez del agua, genera una reducción parcial de color, de la carga bacteriana (Matilainen et al., 2010) y de un cierto rango de virus (Kelderman \& Kruis, 2001).

Actualmente, el coagulante más utilizado para tratamiento del agua superficial es el sulfato de aluminio multihidratado (Solis et al., 2012). Se utilizan diferentes concentraciones de la sal de aluminio que posibilitan la reducción rápida de la turbidez. La Organización Mundial de la Salud (OMS), recomienda el uso de sales de aluminio en función de las diferentes necesidades generadas por las 
diversas calidades de agua cruda, especificándose para plantas pequeñas el uso de hasta 0,2 mg/L (Organización Mundial de la Salud, 2003). El uso de sales de aluminio, ha generado problemas que varían desde el alto costo de operación de las plantas -el costo del uso de aluminio varía entre el $55 \%$ y $60 \%$ del costo total del tratamiento- (Nieves \& Ramón, 2014), hasta la presencia de residuos de aluminio en el agua tratada que representan riesgos para la salud del consumidor (Tostado, 2014).

En el agua, las partículas coloidales están cargadas negativamente, razón por la cual, para su remoción se procede a la adición de iones cargados positivamente como $\mathrm{Al}^{3+}$ con lo que se rompe la repulsión existente entre ellas. Una vez desestabilizadas las partículas comienzan a aglomerarse hasta formar flóculos, que pueden sedimentarse fácilmente. Es en este momento cuando conviene agregar polímeros orgánicos, sintéticos o naturales, para fortalecer la formación de flóculos (Breemen, 2001). En esta etapa del proceso el uso de biopolímeros como la quitina es una opción conveniente, debido a su insolubilidad en agua (Mármol et al., 2011) y su capacidad de producir floculación.

De esta manera, es importante definir el rango de concentración de quitina que permita la mayor disminución de los niveles de turbidez, como consecuencia del aporte del biopolímero a la floculación; así como también definir la posibilidad de que la quitina sea utilizada en las dos etapas de eliminación de los sólidos: coagulación y floculación.

\section{MATERIALES Y MÉTODOS}

Tratamiento preliminar de la muestra

Se limpió manualmente el exoesqueleto de camarón retirando toda la materia orgánica adherida, se lavó con agua destilada tipo I. La muestra fue escaldada por tres minutos y secada en una estufa de convección BINDER a $40{ }^{\circ} \mathrm{C}$, hasta lograr consistencia frágil. El sólido seco se molió y tamizó en malla 20. Se extrajo la fase lipídica con una mezcla ace- 
tona:agua $(9: 1)(\mathrm{v} / \mathrm{v})$, hasta lograr la decoloración total.

\section{Obtención de quitina}

El sólido desengrasado se desmineralizó con agitación constante, a reflujo con ácido clorhídrico en una relación muestra: ácido 1:10 (p/v). Se analizaron los efectos de los factores: tiempo de calentamiento $(\mathrm{t})$, temperatura (T) y concentración de ácido clorhídrico (C), sobre la disminución de la concentración de calcio. Se utilizó un diseño experimental $2^{3}$, con réplica de diseño completo al $95 \%$ de confianza. Los experimentos fueron aleatorizados en el programa JMP Analitical Discovery ${ }^{\circledR}$. Los niveles de los factores de estudio se detallan en la Tabla 1. Para estimar los efectos de los factores sobre las variables respuesta se utilizó el algoritmo de Yates (Box et al., 2005)

Tabla 1. Niveles de los factores de estudio para desmineralización de exoesqueleto de camarón

\begin{tabular}{lccc}
\hline \multirow{2}{*}{ Factores } & \multirow{2}{*}{ Simbología } & \multicolumn{2}{c}{ Niveles } \\
\cline { 3 - 4 } & & $-\mathbf{1}$ & $\mathbf{+ 1}$ \\
\hline Tiempo & $\dagger(\mathrm{min})$ & 30 & 60 \\
\hline Temperatura & $\mathrm{T}\left({ }^{\circ} \mathrm{C}\right)$ & 22 & 25 \\
\hline Concentración $\mathrm{HCl}$ & $\mathrm{C}(\mathrm{eq} / \mathrm{L}$, Normalidad $)$ & 0,5 & 1,0 \\
\hline
\end{tabular}

El análisis del contenido de calcio en las muestras desmineralizadas se realizó por absorción atómica en un espectrofotómetro PERKIN ELMER 100.

Utilizando igual metodología se definieron los efectos sobre el porcen- taje de disminución de proteína en las muestras desmineralizadas. Los parámetros experimentales se definieron utilizando un diseño experimental $2^{2}$ aleatorizado, al $95 \%$ de confianza. Los niveles de los factores de estudio se detallan en la Tabla 2. 
Tabla 2. Niveles de los factores de estudio para desproteinización del exoesqueleto de camarón

\begin{tabular}{lccc}
\hline \multirow{2}{*}{ Factores } & \multirow{2}{*}{ Simbología } & \multicolumn{2}{c}{ Niveles } \\
\cline { 3 - 4 } & & $\mathbf{- 1}$ & $\mathbf{+ 1}$ \\
\hline Tiempo & $\dagger(\mathrm{min})$ & 60 & 120 \\
\hline Concentración $\mathrm{NaOH}$ & $\mathrm{C}(\%)$ & 3,5 & 4,0 \\
\hline
\end{tabular}

La desproteinización se realizó a reflujo con hidróxido de sodio en una relación muestra:base 1:10 (p/v). El contenido de proteína remanente fue analizado mediante el método Kjeldalh. El sólido obtenido se neutralizó por lavados sucesivos con agua y se decoloró con peróxido de hidrógeno $0,1 \%(v / v)$. La quitina obtenida se caracterizó mediante: su solubilidad en agua y en ácido clorhídrico a diferentes concentraciones, el contenido de nitrógeno determinado en un equipo de análisis elemental cuantitativo VARIAN MICROCUBE, las bandas de absorción de infrarrojo obtenidas en un equipo FT-IR JASCO-4600 utilizando un ATR de Zn-Se, el peso molecular fue calculado a partir de mediciones de viscosidad intrínseca del polímero utilizando como solvente ácido clorhídrico 0,3 mol/L - cloruro de sodio $0,2 \mathrm{~mol} / \mathrm{L}$ a una temperatura de $25{ }^{\circ} \mathrm{C}$ (Costa et al., 2015) utilizando un viscosímetro capilar.

\section{Coagulación - Floculación de sólidos en agua.}

A diferencia del tratamiento tradicional, se decidió probar directamente con diversas concentraciones de quitina como biopolímero coagulante y floculante, para luego contrastar los resultados obtenidos con los niveles de reducción de turbidez ocasionada con el uso de sulfato de aluminio, $\mathrm{Al}_{2}\left(\mathrm{SO}_{4}\right)_{3} \cdot 18 \mathrm{H}_{2} \mathrm{O}$. Se determinó la turbidez de una suspensión acuosa de 800 ppm preparada con zeolita comercial molida y tamizada en malla 100, la suspensión se dejó decantar por 12 horas y se utilizó el sobrenadante, con una turbidez de 54 NTU.

Las pruebas de jarras se realizaron en siete equipos de agitación magnética THERMO Scientific. Utilizando un volumen constante de agua preparada, se añadieron volúmenes variables de una solución de 200 ppm de 
quitina en ácido clorhídrico MERCK grado analítico $6 \%(\mathrm{v} / \mathrm{v})$. Se agitó el agua con el biopolímero, por un minuto a $360 \mathrm{rpm}$ y por 20 minutos a 60 rpm; se dejó en reposo por 30 minutos y se midió la turbidez de las muestras de agua tratadas en un turbidímetro HACH-2100Q. Se calculó la disminución de turbidez del agua preparada, contrastada con el blanco constituido por el sobrenadante del agua preparada con una turbidez de 54 NTU. Igual metodología se utilizó para el tratamiento del agua, con sulfato de aluminio grado analítico $\left(\mathrm{Al}_{2}\left(\mathrm{SO}_{4}\right)_{3} .18 \mathrm{H}_{2} \mathrm{O}\right.$ Fisher Scientifc).

\section{RESULTADOS}

\section{Obtención de quitina}

La desmineralización se verificó mediante la disminución de calcio. El análisis del diseño experimental definió que a $22{ }^{\circ} \mathrm{C}$, con un tiempo de reflujo de 30 minutos y utilizando $\mathrm{HCl} 1 \mathrm{~N}$, se obtiene el mayor porcentaje de desmineralización, con una disminución de calcio respecto del contenido original del exoesqueleto de camarón de 97,80 \% (p/p).

En el caso de la desproteinización, las variables analizadas, así como su interacción no tienen significancia estadística; por tanto, se utilizaron los niveles más bajos de los factores 3,5 $\%$ (p/v) de $\mathrm{NaOH}$ y 60 min de reflujo, con una disminución del porcentaje de proteína de $(32,18 \pm 0,14 \%$, respecto del contenido original.

La quitina se obtuvo como un sólido de color blanco en forma de láminas, insoluble en agua y en ácido clorhídrico diluido, soluble en ácido clorhídrico concentrado y con un peso molecular de 202,978 $\pm 1,235 \mathrm{~g} / \mathrm{mol}$. El espectro infrarrojo para la quitina obtenida experimentalmente, se presenta en la Figura 2. 


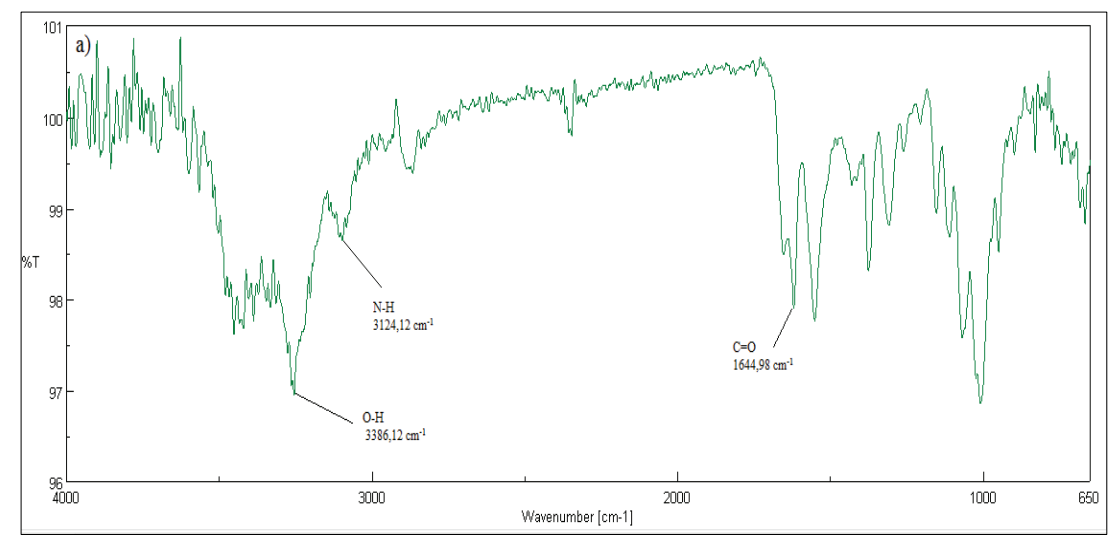

Figura 2. Espectro infrarrojo de quitina

Coagulación - Floculación de sólidos en agua.

Los resultados de las pruebas de jarras, se presentan en la Tabla 3, y están definidos como el cambio en la turbidez contrastada con el blanco; considerando que en el blanco (descrito en la metodología), únicamente se producen efectos de sedimentación de sólidos debido a la gravedad y al proceso de agitación.

\section{DISCUSIÓN}

Con la aplicación de diseño experimental detallado y la selección de las condiciones experimentales, se obtuvieron valores promedio para desmineralización y desproteinización del exoesqueleto de camarón 97,80 \pm $3,13 \%$ (p/p) y $32,18 \pm 0,14 \%(p / p)$, respectivamente; con un porcentaje de rendimiento de quitina de $27,50 \%$ $(\mathrm{p} / \mathrm{p})$; mayor que el que obtuvieron
Mármol et al (2011), que fue de $24,06 \%$ (p/p).

El efecto diferencial en la coagulación -floculación de sólidos en el agua, se explica por el uso de productos de diferente naturaleza (uno de ellos el biopolímero), pero a la misma concentración. Los resultados obtenidos tanto para el sulfato de alu- 
minio como para la quitina, se aprecian como el cambio de la turbidez (NTU), como se registra en la tercera y quinta columna de la Tabla 3. Se puede apreciar también como a una misma concentración se obtienen cambios positivos o negativos según se trata de la aplicación de sulfato de aluminio o de quitina, como se registra para las determinaciones dos $y$ tres en la Tabla 3.

Tabla 3. Cambios de turbidez de agua tratada con sulfato de aluminio y quitina

\begin{tabular}{ccccc}
\hline Determinación & $\begin{array}{c}\text { Conc. } \\
\mathrm{Al}_{2}\left(\mathrm{SO}_{4}\right)_{3} \mathbf{1 8 H _ { 2 } \mathbf { O }} \\
(\mathbf{p p m})\end{array}$ & $\begin{array}{c}\text { Cambio de } \\
\text { turbidez } \\
(\mathbf{N T U})\end{array}$ & $\begin{array}{c}\text { Conc. } \\
\text { Quitina } \\
(\mathbf{p p m})\end{array}$ & $\begin{array}{c}\text { Cambio de } \\
\text { turbidez } \\
(\text { (NTU) }\end{array}$ \\
\hline $\mathbf{1}$ & 0,1755 & 1,00 & 0,1755 & 4,10 \\
$\mathbf{2}$ & 0,3510 & $-12,00$ & 0,3510 & 27,50 \\
$\mathbf{3}$ & 0,5265 & $-18,00$ & 0,5265 & 27,23 \\
$\mathbf{4}$ & 0,7020 & 2,00 & 0,7020 & 24,25 \\
$\mathbf{5}$ & 0,8775 & 13,00 & 0,8775 & 22,50 \\
$\mathbf{6}$ & 10,530 & 6,00 & 10,530 & 31,10 \\
\hline
\end{tabular}

De acuerdo a su estructura química, la quitina presenta solubilidad en ácido clorhídrico concentrado debido a la presencia de nitrógenos amídicos en los cuales, la resonancia de los electrones libres del nitrógeno, con el oxígeno del grupo acetilo disminuye las características básicas de éste (Wade, 2012). El peso molecular obtenido para el biopolímero se calculó con la ecuación de Mark Houwink-Sakurada (Costa el al., 2015) y es menor que el reportado por otros autores (Cortizo \& Cortizo, 2011), lo cual se puede explicar por la variabilidad de las condiciones de obtención del biopolímero y el solvente utilizado en las determinaciones de viscosidad. El espectro infrarrojo de la Figura 2, define la presencia de bandas de estiramiento de los enlaces $-\mathrm{OH}$ a $3386 \mathrm{~cm}^{-1},-\mathrm{NH}$ a 3124 $\mathrm{cm}^{-1},-\mathrm{C}=\mathrm{O}$ a $1644 \mathrm{~cm}^{-1}$ además de las absorciones características de enlaces $-\mathrm{C}_{\mathrm{sp} 3} \mathrm{H}$ de la cadena carbonada (Wade, 2012), que coinciden con la estructura de la unidad repetitiva del biopolímero (Figura 1) (Velás- 
quez, 2003) y son comparables con las del espectro infrarrojo de quitina reportado por Ramirez et al (2016) donde las bandas de absorción se presentan a: $-\mathrm{OH}$ a $3436 \mathrm{~cm}^{-1}, \mathrm{NH}$ a $3198 \mathrm{~cm}^{-1},-\mathrm{C}=\mathrm{O}$ a $1661 \mathrm{~cm}^{-1}$.

Se definió una metodología experimental en la que se estableció que, de acuerdo a los valores de los efectos, el porcentaje de disminución de calcio se ve afectado fundamentalmente por los cambios de concentración del ácido clorhídrico utilizado, teniéndose un decremento del porcentaje de calcio de -2,16 unidades cuando la concentración de ácido cambia de -1 a +1 en la zona experimental. Las variables tiempo, temperatura y su interacción provocan efectos de incremento en el porcentaje de calcio. De acuerdo al planteamiento de Montgomery \& Ruger (2002), estos resultados influyen directamente sobre la variable respuesta.

Los resultados para desproteinización indicaron que las variables tiempo $(\mathrm{t})$ y concentración de hidróxido de sodio (C) no tienen significancia estadística en la zona experimental analizada, por lo que se seleccionaron para la implementación de la me- todología los valores de concentración y tiempo más bajos.

La definición de la concentración de quitina óptima para la coagulación floculación de sólidos en aguas de baja turbidez, debe definirse en función de la reducción elevada en la turbidez a bajas concentraciones de quitina, considerando la importancia económica que se deriva del uso de menor cantidad de biopolímero para producir este efecto. Por tanto, se utilizaron concentraciones de quitina entre 0,15-1,00 ppm.

De acuerdo a la Figura 3, se puede establecer que los cambios de turbidez en función de la concentración de quitina en el agua analizada, tienen un comportamiento no lineal, debido a la baja correlación existente entre las variables $(R=0,650)$. Se observa que a bajas concentraciones de quitina (entre 0,15 - 0,30 ppm), se tiene un efecto brusco en la disminución de la turbidez del agua, el mismo que tiene un comportamiento cambiante a medida que crece la concentración de biopolímero, explicable debido a que a más altas concentraciones de biopolímero, la capacidad de coagulación es menor. 
El punto mínimo de la curva que re- $\quad$ bidez, se encuentra entre 0,30 - 0,45 presenta el valor más bajo para la tur- ppm de quitina.

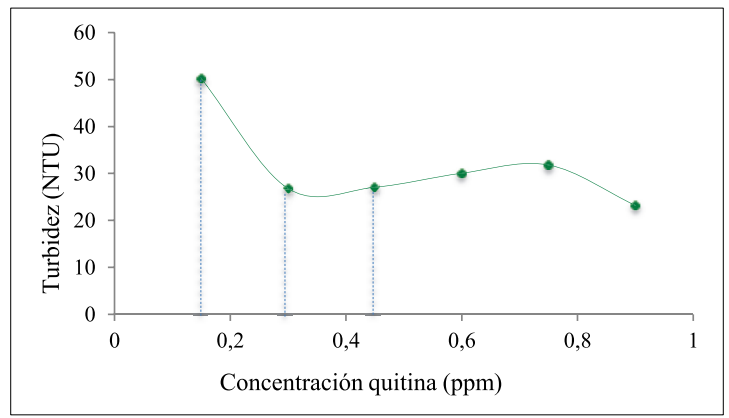

Figura 3. Turbidez del agua tratada con quitina a diferentes concentraciones

Para evidenciar el punto mínimo de la curva presentada en la Figura 3, se graficó la reducción de turbidez respecto del blanco vs la concentración de quitina entre 0,15 - 0,45 ppm, que se presenta en la Figura 4. Así, se obtiene una curva que se ajusta a una regresión polinomial de segundo gra- do, con un coeficiente de correlación $R=1$, en la cual se puede observar que la máxima reducción en la turbidez se produce cuando se utiliza concentraciones de quitina entre 0,35 - 0,40 ppm, zona de los máximos de la curva (Figura 4).

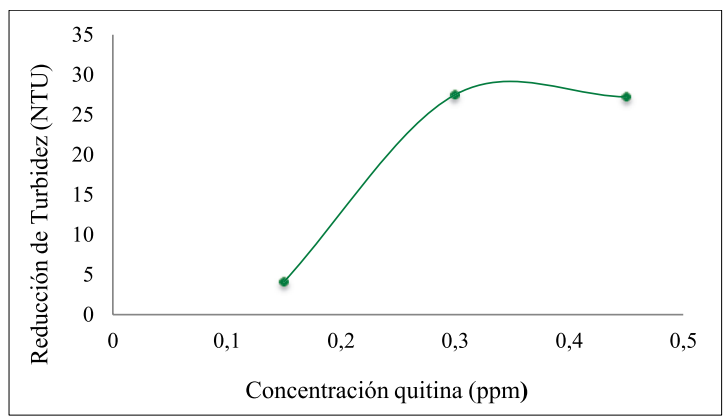

Figura 4. Reducción de turbidez del agua tratada con quitina a diferentes concentraciones 
Este resultado es comparable con lo observado en la literatura técnica internacional disponible, la misma que registra que la dosis óptima, para tratamiento de agua para consumo humano con quitina, se ubica alrededor de los 40 ppm, y que los resultados obtenidos después del tratamiento con quitina a la dosis óptima mencionada reflejan valores de turbidez entre 0,39 y 0,69 NTU (Fuentes et al., 2018).

Por otro lado, a partir de los datos obtenidos en esta investigación, se pudo determinar mediante cálculo teórico, que cuando la concentración de quitina es 0,37 ppm, se alcanza la má- xima reducción en la turbidez del agua analizada, hasta 30,32 NTU. Sin embargo, si se analiza el comportamiento del sulfato de aluminio (Figura 5) en el mismo rango de concentraciones que se estableció para quitina (Figura 3), se puede observar que en el rango entre $0,15-0,60$ ppm, los valores en la reducción de la turbidez son negativos, notándose que la turbidez del agua se incrementa en este segmento de la curva; efecto contrario al observado para quitina. Solamente se tiene un efecto de reducción en la turbidez del agua cuando las concentraciones de sulfato de aluminio superan las 0,70 ppm.

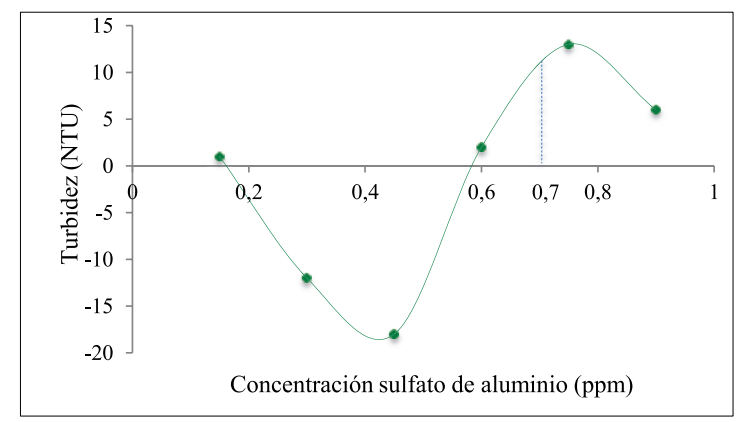

Figura 5. Turbidez del agua tratada con sulfato de aluminio a diferentes concentraciones 
De esto se desprende que el ajuste polinomial de segundo grado para sulfato de aluminio en el rango de concentración (0,15 - 0,45 ppm) utilizado para quitina, no presenta utilidad en la disminución de la turbidez, ya que se tiene una curva con valores negativos. Sin embargo, conforme aumenta la concentración, esta gráfica se interpreta como una sucesión de incrementos progresivos de turbidez hasta alcanzar los valores que se encuentran cercanos al rango entre 0,4-0,5 ppm de quitina.
Además, el ajuste polinomial presenta una baja correlación ( $R=0,930)$.

Por otro lado, mientras que para quitina el rango de concentración donde se evidencia la mayor disminución de turbidez, se encuentra entre 0,15 - 0,45 ppm; con sulfato de aluminio para este mismo rango de concentración se obtiene una función negativa que representa el incremento de turbidez, como puede observarse en la Figura 6.

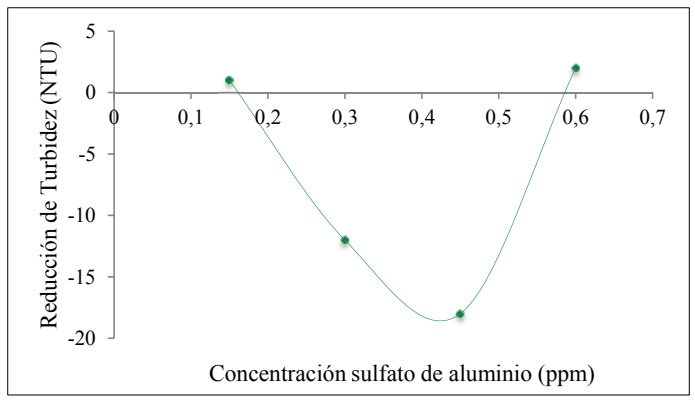

Figura 6. Reducción de turbidez del agua tratada con sulfato de aluminio a diferentes concentraciones

Con concentraciones experimentales entre 0,15 - 0,45 ppm de quitina, se observan descensos en la turbidez que de acuerdo a los cálculos teóri- cos se encuentran entre 7,55 - 50,27 $\%$; teniéndose el valor máximo de $55,84 \%$ en el descenso de turbidez con 0,37 ppm de quitina. 
Con sulfato de aluminio, se tiene el efecto de descenso de turbidez cuando su concentración supera las 0,70 ppm, es decir la concentración eficaz de sulfato de aluminio duplica las de quitina, para generar el mismo efecto de reducción de turbidez. En el rango de 0,15 - 0,45 ppm la quitina alcanza la mayor disminución de turbidez, mientras que el sulfato de aluminio produce el efecto inverso es decir ocasiona el incremento de la turbidez.

\section{CONCLUSIÓN}

Se obtuvo quitina por decoloración, desmineralización y desproteinización del exoesqueleto de camarón. El biopolímero obtenido se comporta como coagulante-floculante de sólidos suspendidos en agua, produ- ciendo reducción en los niveles de turbidez, característica que la convierte en una alternativa válida para disminuir el uso de compuestos de probada toxicidad en el tratamiento de agua para consumo humano.

\section{LISTA DE REFERENCIAS}

Breemen, V. (2001). Water Treatment Conventional an advance Treatmet Methods. Holanda: International Institute for Infrastructural, Hidraulic and Environmental Engineering.

Box, G., Hunter, J., \& Hunter, W. (2005). Statistics for experiments design, innovation and discovery. New Jersey, USA: Wiley Interscience.

Costa, C., Teixeira, V., Delpech, M., Souza, J., \& Costa, M. (2015). Viscometric study of chitosan solutions in acetic acid/sodium acetate and acetic acid/sodium chloride. Carbohydrate Polymers, 245-250. 
Cortizo, M. S., \& Cortizo, A. M. (2011). Desarrollo y caracterización de matrices compuestas quitosano/polímero sintético para regeneración de tejido óseo. Universidad de La Plata, Ciencias Biológicas. La Plata: Universidad de la Plata.

Fuentes, L., Mendoza, I., Chávez, A., \& Cedeño, O., (2018). Evaluación de quitina como coagulante para potabilización de aguas con alta turbidez. Impacto Científico, 12 (1)

Kelderman, \& Kruis. (2001). Laboratory Course Aquatic Chemistry and its Aplications in Enviromental Engineering. Holland: Enviromental Enginering International Institute for Infrastructural Hydraulic and Enviromental Engineering.

López, M. A. (2012). Obtención y caracterización de quitosanos modificados: ingredientes funcionales con aplicaciones tecnológicas y biológicas en la industria alimentaria. Tesis Doctoral, Universidad Complutense de Madrid, Departamento de Nutrición, Bromatología y Tecnología de los Alimentos, Madrid.

Mármol, Z., Páez, G., Rincón, M., Araujo, K., Aiello, C. C., \& Gutiérrez, C. (2011). Quitina y Quitosano polímeros amigables. Una revisión de sus aplicaciones. Revista Tecnocientífica URu, 53 - 57.

Matilainen, A., Vepsäläinen, M., \& Sillanpää, M. (2010). Eliminación de materia orgánica natural por coagulación durante el tratamiento de agua potable: una revisión. Avances en coloides y ciencia de interfaz, 159 (2).

Montgomery, D., \& Ruger, G. (2002). Applied Statistics and Probability for Engineers. The USA: John Wiley \& Sons, Inc.

Nieves, T., \& Ramón, J. (2014). Análisis del costo de producción de agua potable y de agua por pérdidas no contabilizadas en las plantas de producción de Tomebamba y Machángara. Tesis Maestría, Universidad Politécnica Salesiana, Cuenca.

Organización Mundial de la Salud. (2003). Aluminium in drinking water. Guias de la OMS para la calidad del agua potable. Ginebra. 
Ramirez, A., Benítez, J., Rojas de Astudillo, L., \& Gascué, B. (2016). Polymers materials type hydrogels: Review of their characterization by FTIR, DSC, SEM and TEM. Revista Latinoamericana de Metalurgia y Materiales.

Restrepo, A., \& Guarín, S. (2004). Valorización de residuos. Universidad de Antioquia, Escuela de Ingeniería Ambiental, Envigado.

Solis, R., Laines, J., \& Hernandez, J. (2012). Mezclas con potencial coagulante para clarificar aguas superficiales. Revista Interamericana de Contaminación Ambiental (28), $229-236$.

Tostado, E. (2014). Neurotoxicidad de los metales pesados: plomo, mercurio y aluminio. Tesis maestría, Universidad de Valladolid, Facultad de Medicina, Valladolid.

Velásquez, C. L. (2003). Algunos usos del quitosano en sistemas acuosos. Revista Iberoamericana de Polímeros, 94.

Wade, L. (2012). Química Orgánica (Vol. 2). México, México: PEARSON. 